\title{
X-RAY SPECTRA OF YOUNG SUPERNOVA REMNANTS
}

\author{
S.S. Holt \\ Laboratory for High Energy Astrophysics \\ NASA/Goddard Space Flight Center \\ Greenbelt, Maryland 20771
}

\section{A. HISTORICAL NOTES}

The early identification of the strong $X$-ray source in Taurus with the Crab nebula (Bowyer et al. 1964) was the first milestone in the association of X-ray emission with supernova remnants. Unfortunately, it proved to be "red herring" which clouded the interpretation of X-ray emission from supernova remnants for a decade. Because the Crab was one of the brightest $X$-ray sources in the sky at a few keV, the interrogation energy of the early surveys, and because it was the first (and for several years the only) X-ray source conclusively identified, the potential association of a supernova origin with the larqe body of unidentified $X$-ray sources was not an unreasonable hypothesis.

By 1970 proportional counter spectra from rocket-borne observations, with resolving power $E / \Delta E<2$, were available for Cas $A$ and Tycho as well as the Crab (Gorenstein, Kellogg and Gursky 1970). Since the statistical significance of the data points were exposure-limited, they were fittable with either bremsstrahlung continua with temperatures of $\sim 10^{7} \mathrm{~K}$ or power-law spectra; the analoay with the Crab suggested to many interpreters of the data that the latter representations of the spectra were more appropriate. By that time the Crab spectrum was well-measured to be a power-law with $\alpha \sim 1$ between 1-100 keV, and relatively well-understood (from the point of view of available energy, if not energy transfer) as arising from the non-thermal conversion of rotational energy from its pulsar. Similar pulsars which were unobservable because of their beaming characteristics were suggested for other young supernova remnants, since only young pulsars could provide the required rotational eneray loss (e.g. Pacini 1971). Similarly, models featuring hidden pulsars in sources not associated with traditional supernova remnants (e.g. Davidson, Pacini and Salpeter 1971) were relatively popular.

The discovery of "X-ray pulsars" (Giacconi et al. 1971), distinguishable from radio pulsars like the Crab by their inability to account for the source luminosity from rotational eneray loss, provided the key to understanding the energy generation mechanism of the non-SNR 
sources in terms of mass accretion onto neutron star components of binary systems, as had been suggested by Shklovsky (1967). But what about the young SNRs Cas A and Tycho? An early suggestion by Heiles (1964), that the SN blast wave sweeping up the interstellar medium into a shell of shocked material would provide an observable X-ray source, seemed more appropriate to older remnants in which enough mass had been swept up to provide an adequate emission measure, and enough time had elapsed to allow the radiating shell to equilibrate at a temperature low enough to cool efficiently (e.g. Woltjer 1972). Nevertheless, a blast wave interpretation for the young SNRs increased in popularity as the newer data indicated better agreement with a thermal spectrum. The key experimental result was the discovery of Fe line emission from Cas $A$ (Serlemitsos et al. 1973) which, although originally interpreted by its discoverers as arising from charge exchange with $\sim 10 \mathrm{MeV} /$ nucleon cosmic rays, was quickly recognized as the signature of thermal processes in the remnant. As data from satellite experiments became available (Charles et al. 1975; Pravdo et al. 1976; Davison, Culhane and Mitchell 1976) this picture was refined to require two separate thermal components for consistency with these higher statistical precision data. Itoh (1977) suggested that the harder of these two components was associated with the biast wave, while the softer was associated with a thin, dense shell of ejecta accumulated from the "reverse shock" postulated by McKee (1974). The history of Cas A and Tycho measurements in the decade preceding the first spectral data from Einstein is aiven in Table 1.

Table 1

EARLY THERMAL FITS TO CAS A AND TYCHO

\begin{tabular}{|c|c|c|c|c|}
\hline \multirow[b]{2}{*}{$\operatorname{Ref}$} & \multicolumn{2}{|c|}{ Cas A } & \multicolumn{2}{|c|}{ Tycho } \\
\hline & Tlow & Thigh & Tlow & $T_{\text {high }}$ \\
\hline al. 1970 & $\begin{array}{l}---- \\
.83 \pm .06\end{array}$ & $\begin{array}{c}1.25 \pm .34 \\
-\end{array}$ & $.45 \pm .05$ & 2. $2 \pm 1.0$ \\
\hline $\begin{array}{l}1975 \\
\text { al. } 1975 \\
\text { al. } 1976 \\
\text { al. } 1976\end{array}$ & $\begin{array}{l}.73 \pm .20 \\
.7 \\
1.08 \pm .03\end{array}$ & $\begin{array}{l}2.3 \\
2.6 \pm 1.0 \\
3.9 \pm .6 \\
5.2 \pm .6\end{array}$ &.$\overline{-.-}$ & $\begin{array}{l}1.9 \\
-.-- \\
3.5 \pm .3\end{array}$ \\
\hline
\end{tabular}

* refined analysis of data of Serlemitsos et al. (1973)

\section{B. THE EINSTEIN SSS DATA}

The newer Einstein data summarized here are from the Solid State Spectrometer (SSS), a cryogenically-cooled Si(Li) device with a FWHM energy resolution of $\sim 160 \mathrm{eV}$ over its effective 0.5-4.5 keV energy range (see Holt et al. 1979 for experiment details). The spectra I shail discuss have already been preliminarily reported in the i iterature. The new information presented here will be of two kinds: a 
refined set of experimental spectra with all sources of background over the full 0.5-4.5 keV band now properly accommodated, and some remarks about the progress which has been made in the physical modelling of SNR to reproduce these spectra.

A Crab spectrum is presented in Figure 1 ; it represents one of 100 such spectra obtained over the 1 ifetime of the experiment, all of which were used for calibration purposes to ensure uniformity in the interpretation of detector response with time. The input spectrum is presumed to be a featureless power law of index $\alpha=1.1$ observed through an interstellar medium of cosmic abundance with column density $\mathrm{N}_{\mathrm{H}}=3 \times 10^{21}$ atoms $\mathrm{cm}^{-2}$. Note that the experiment introduces an apparent kink in the spectrum at $\sim 0.8 \mathrm{keV}$ (arising from the nickel with which the telescope is coated), but that any other features

(particularly ones assocaited with silicon, of which the detector is made, and which might be expected near $1.8 \mathrm{keV}$ ) are much smaller than the statistical precision of one of the displayed pulse-height channels in a typical measurement. The Crab spectrum is illustrative, therefore, of the fact that any potential line features in the spectra are not systematically induced in the detector.

The line features which are observed in young SNR spectra are indicated in Figure 2, where are plotted the pulse height data from an exposure to the Tycho (SN 1572) remnant, together with a comparison spectrum for illustrative purposes. The comparison spectrum is that expected from a $T=0.5 \mathrm{keV}$ solar-abundance plasma in thermal equilibrium, viewed through an interstellar column density of $2 \times 10^{21}$ atoms $\mathrm{cm}^{-2}$; this comparison histogram is not convolved through the detector response, but is smeared to a FWHM resolution of $\sim 50 \mathrm{eV}$ with exactly the same bin widths as the experimental data. The shaded portion of the comparison spectra is the contribution from Fe-L emission from the isothermal plasma, and the darkened portion the contribution from $\mathrm{Si}-\mathrm{K}$ emission; the four blackened bumps represent $\mathrm{Si}+12 \mathrm{~K} \alpha, \mathrm{Si}+13 \mathrm{~K}, \mathrm{Si}^{+12} \mathrm{~K}_{\beta}$ and $\mathrm{Si}^{+12} \mathrm{~K}_{\gamma}$ in order of increasing eneray. From the obvious line identifications, therefore, it is clear that the observed lines are characteristic of a relatively low temperature $(\mathrm{e} . \mathrm{g} . \mathrm{T}=0.5 \mathrm{keV}) \mathrm{plasma}$, in the sense that the helium-like lines of the $Z>10$ constituents are evident while the hydrogen-like lines are not, but the equivalent continuum widths of the observed lines are considerably in excess of the equilibrium values for solar abundances. Most of the remainder of this paper will be devoted to a discussion of the modelling of such spectra.

\section{SPECTRAL MODELLING}

Figure 1 displays the SSS spectrum from Cas A. As originally reported by Becker et a1. (1979), the data cannot be fitted with a single equilibrium temperature with any abundance distribution; instead, it can be reasonably well-fitted with two equilibrium components. The lines are consistent with an equilibrium plasma at $T \simeq 0.65 \mathrm{keV}$ (albeit 


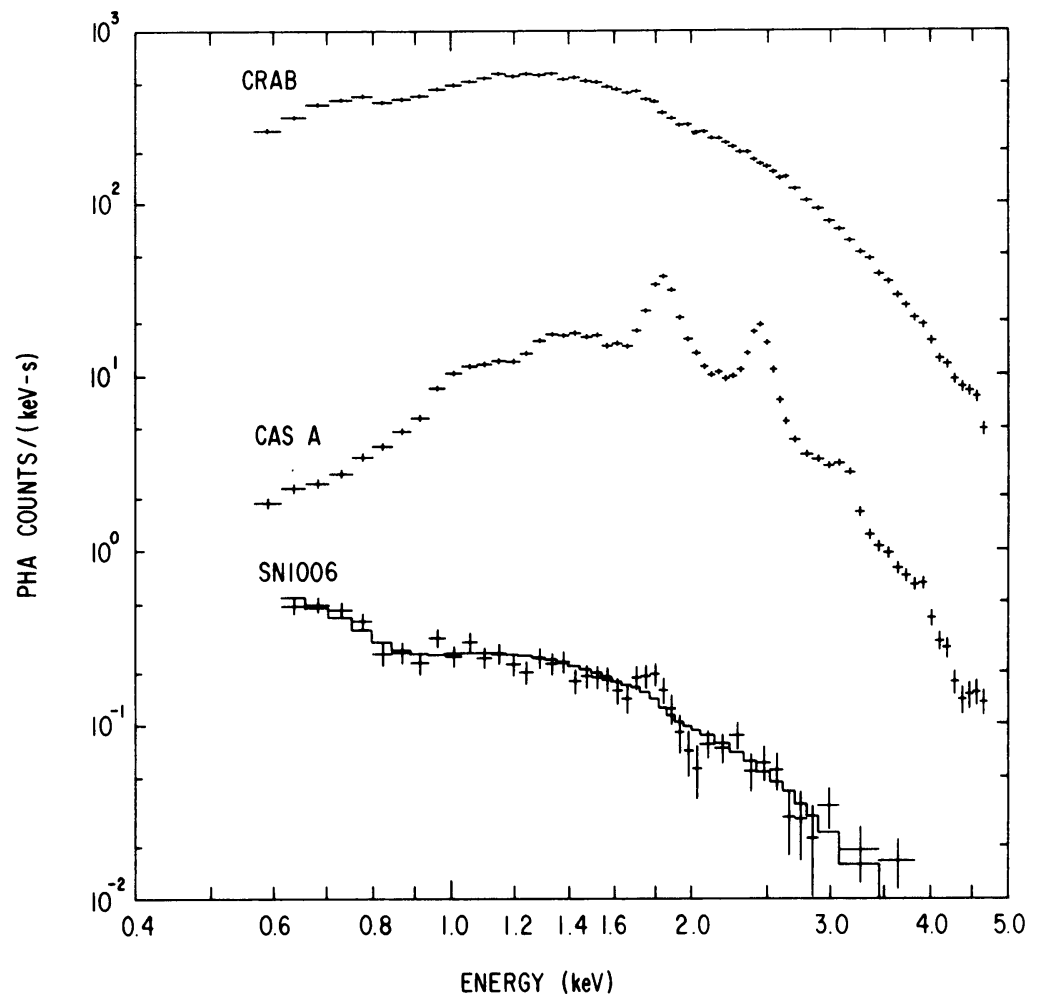

Figure 1. Experimental SSS spectra for the Crab nebula, CasA and SN1006. The solid histogram through the SN1006 data points represents a power law of index $\alpha=1.2$ observed through a column density of $9 \times 1020$ atoms $\mathrm{cm}^{-2}$.

with overabundances in the line-emitting components), but a higher temperature component is required to replicate the harder continuum observed and to smoothly connect to the higher eneray measurements; because the bremsstrahlung continua from any temperatures $\approx 3 \mathrm{keV}$ are indistinguishable in the SSS 0.5-4.5 keV bandpass, a fixed $4 \mathrm{keV}$ was chosen to approximately connect to the higher energy measurements.

The data presented in Figure 1 are somewhat more refined than originally presented in Becker et al. (1979), but the important source-fitting parameters are not substantialiy changed. In particular, the best-fit low temperature component (for a 2-temperature equilibrium fit) is still $\mathrm{T} \simeq .65 \mathrm{keV}$, and the Si abundance is $\sim 1.5$ times solar, as determined from the simultaneous fitting of the lines and the continuum. Relative to this inferred Si abundance, the inferred abundances of other important constituents relative to solar proportions are S/Si( 2), Ar/Si( 4), Ca/Si( 2) and Ma/Si( .1). The only significant difference between the results of the present modelling and that originally reported is in the $\mathrm{Fe}$ abundance, as the model extension to 


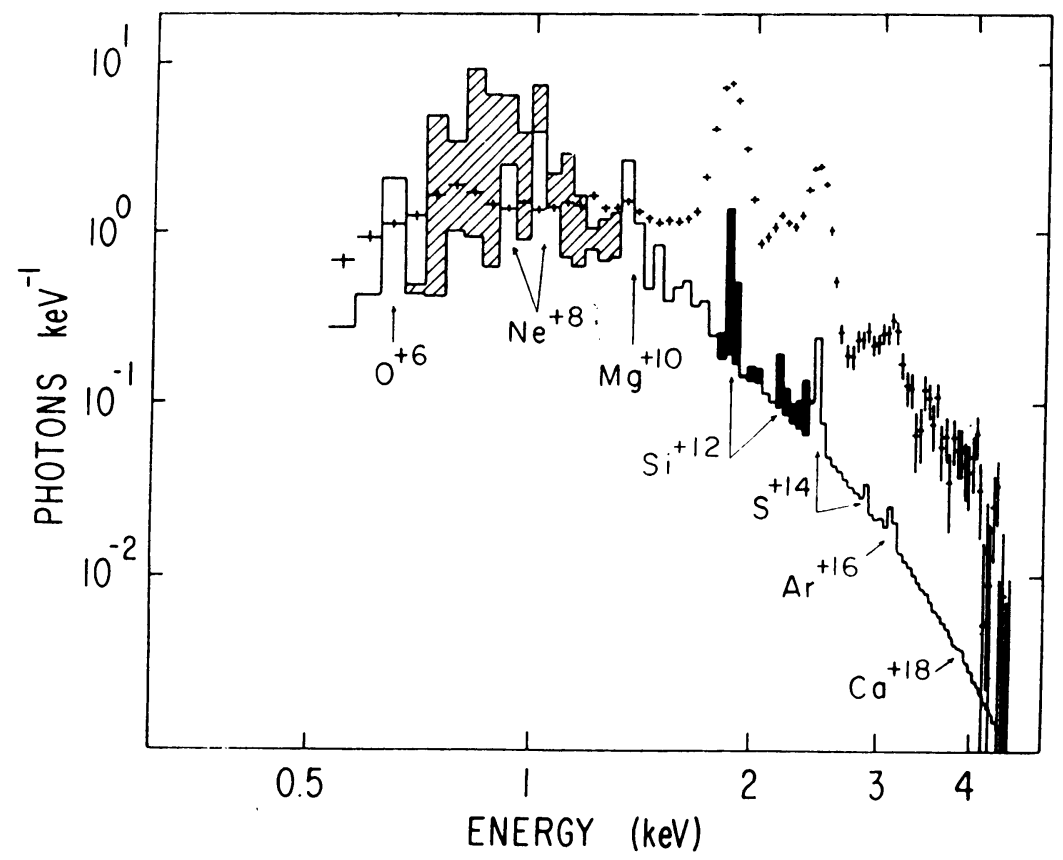

Figure 2. Experimental SSS spectrum of the Tycho SNR, compared with the expected spectrum from a solar-abundance equilibrium plasma at $T=0.5$ $\mathrm{keV}$ as it would be observed with a detection system which has an energy-independent response (see text for additional details).

lower energies changes what was previously a measured $\mathrm{Fe} / \mathrm{Si} \sim .5$, relative to solar, to an upper limit. The lack of a pronounced increase. with $\mathrm{Z}$ in the relative overabundances of $\mathrm{Si}, \mathrm{S}, \mathrm{Ar}$ and $\mathrm{Ca}$ suggests that the standard qualifications regarding non-equilibrium ionization may obscure the important qualitative conclusions that may be drawn from the spectrum. If, as suggested by Fabian et a1. (1980) from an interpretation of the $X$-ray morphology, the remnant is in the free expansion phase and the low energy $X$-ray emission is dominated by the ejecta, the clumpiness of the emission suggests a situation closer to equilibrium (at least within the clumps) than would a smoother distribution, as the Rayleigh-Taylor-induced density enhancements should equilibrate more rapidly than would the unclumped ejecta (see Chevalier 1982 for a discussion of the unclumped density distribution which might be expected from a system like Cas A with pre-SN mass 10ss). The modest abundance enhancement of Si-group material implied by the equilibrium simplification may, therefore, better characterize the spectrum than would a detailed (non-unique) calculation, at least until such time as measurements with better spectral and spatial resolution are available.

In contrast to Cas $A$, the three Type I remnants Tycho, Kepler and SN1006 display a clear necessity for non-equilibrium effects. The spectra of Tycho and Kepler bear marked similarities to each other (see 
Figure 3$)$; utilizing the same two-temperature equilibrium model as a starting point, both have low temperatures of $T \simeq .5 \mathrm{keV}$. Tycho requires a Si abundance which is approximately an order of magnitude in excess of solar (Kepler requires about half the Tycho Si abundance), and for both remnants the allowable relative abundances of $\mathrm{S}, \mathrm{Ar}$ and $\mathrm{Ca}$ increase with atomic number (in the newer equilibrium fits, these implied abundances are even higher than those in Becker et al. 1980a and Becker et al. 1980b), in accordance with the expectation for a non-equilibrium plasma which is still ionizing. The application of a Sedov approximation (Shul1 1983; Hamilton, Sarazin and Chevalier 1983)

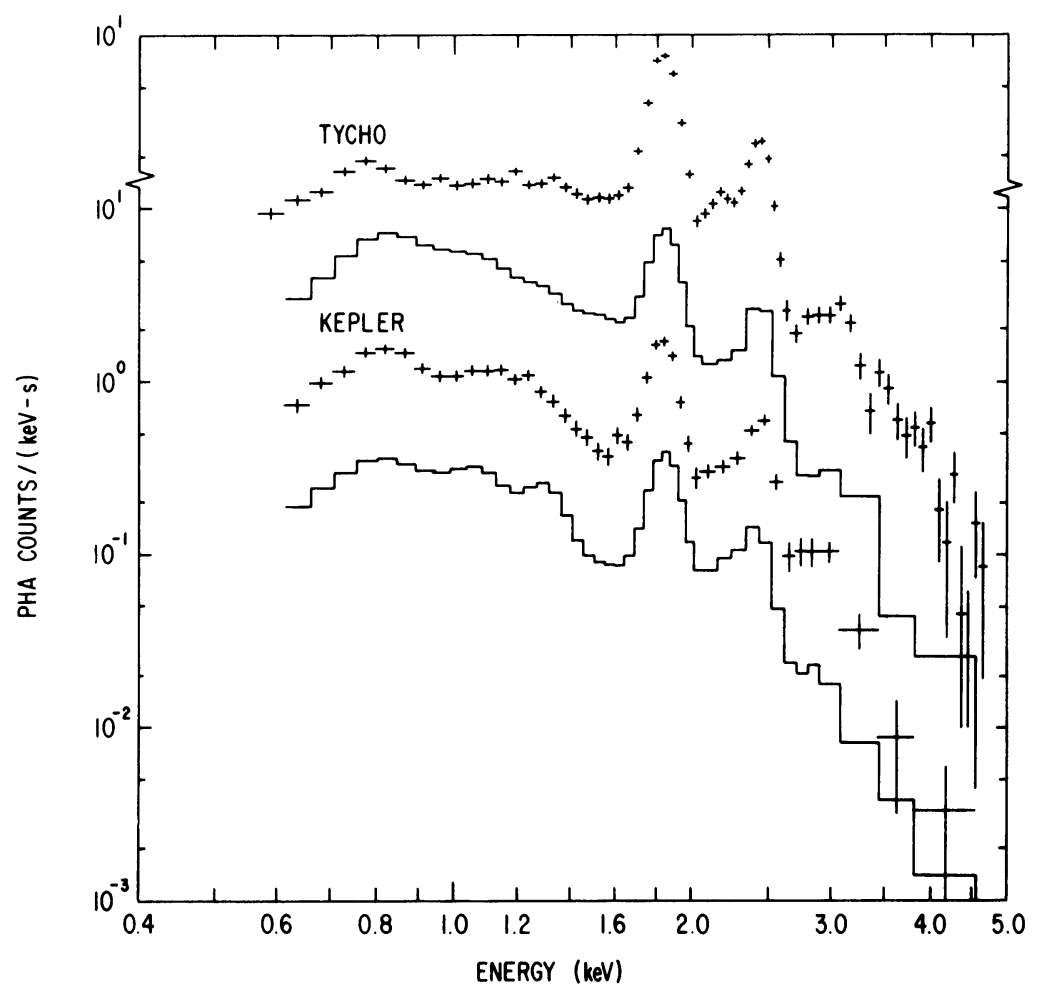

Figure 3 - Experimental SSS spectra of the Tycho and Kepler SNRs. The solid histograms, arbitrarily placed above and below the Kepler spectrum, represent different fits to Kepler of comparable statistical adequacy. The upper fit is a two-temperature equilibrium model for which the abundances are free parameters (see text), while the lower fit is a Sedov model with similarly unconstrained abundances; many such models (with differing $n$, electron fitted with comparable statistical adequacy, but all require $S_{j}$ overabundances relative to solar. 
relieves this latter problem, but its applicability may be problematic. A variety of Sedov models with and without clumping (and with and without the assumption of rapid equilibration of the electrons with the shock) all yield pronounced overabundances of Si, comparable to those found in the equilibrium fits. The relative abundance ratios for the higher-z constituents (i.e. those which are identifyable by their $\mathrm{K}$-emission) are no longer unreasonably high, however; $\mathrm{S} / \mathrm{Si}$ is still $\sim 2$ (as it appears to be for all SNR fits), but $\mathrm{Ar} / \mathrm{Si}$ and $\mathrm{Ca} / \mathrm{Si}$ need not be much higher than unity (i.e. solar). The utilization of a Sedov model (properly constrained to the observable luminosity at the best-estimated distance, with reasonable values of $n=E_{0} n_{0}{ }^{2}$ ) is questionable a priori if the interpretation of the $X$-ray morphology given by Seward, Gorenstein and Tucker (1983) is correct, i.e. if the large fraction of the $X$-ray luminosity arises from clumped reverse-shocked ejecta rather than from the blast wave. Interestingly, Hamilton (this volume) has demonstrated that the emission from the ejecta can be modelled with a Sedov approximation (where the parameter $n$ is a bit more obscure), allowing fits which better match the data in detail (Sarazin, Hamilton and Chevalier, this volume).

A continuing feature of all the fits is the lack of marked overabundances of $\mathrm{Fe}$, as might be inferred from its L-emission (see Figure 2) in accordance with current models of Type I SN which release a large fraction of a solar mass of Fe-group material. The primary difference between the Tycho and Kepler spectra of Figure 3 is in the Fe L-emission; $\mathrm{Fe}$ is virtually undetectable from Tycho, but is required at about the level $\mathrm{Fe} / \mathrm{Si} \sim 1$ (relative to solar) in the various equilibrium and non-equilibrium Kepler fits. Even in Kepler, this abundance is at least an order of magnitude below that expected if the Fe-group material is well-mixed into the X-ray-emitting plasma. Clearly, the retention of the Fe-rich model requires the not-unreasonable assumption that the heavier ejecta is interior to the Si-group ejecta, and has not yet been significantly reverse-shocked.

The X-ray spectrum of SN1006 is even more puzzling. Unlike the younger Type I remnants discussed above, there is no statistically significant evidence for $K-l i n e$ emission. In fact, the $X$-ray spectrum can be fit with a Crab-like power law $(\alpha \sim 1)$ from $0.5 \mathrm{keV}$ out to at least $20 \mathrm{keV}$ (Becker et al. 1980 c). It is worth noting that the higher energy $X$-ray spectra of other SNR can be relatively well-fit by power law approximations, but with spectral indices which are much larger (i.e. the younger and more initially energetic SNR Cas A can be fit with $\alpha \sim 3$, while Tycho can be fit with $\alpha \sim 4$; see Figures 1 and 2 of Pravdo and Smith 1979). Fabian, Stewart and Brinkmann (1982) have suggested that the appearance of a featureless power law may arise from the superposition of emission from differing thermal shells. If the intermediate- $Z$ metals are substantially locked in grains, and if the reverse-shock has long-since passed through the Fe-rich ejecta (so that the remnant interior has cooled to $\left.\sim 10^{6} \mathrm{~K}\right)$, the absence of the prominent lines observed in the younger remnants may be reconciled. It should be noted, however, that the spectral hardness of the high enerqy continuum may be more easily explained with non-thermal processes, e.q. 
synchrotron radiation from electrons accelerated near the blast wave (Reynolds and Chevalier 1981) or possibly even from the naive picture of a hidden pulsar which was so popular ten years ago.

D. FINAL REMARKS

The combination of higher-energy ( $>5 \mathrm{keV}$ ) spectral data from pre-Einstein investigations (see Pravdo, this volume) with morphological and spectral data from all the Einstein instrumentation provides a powerful tool for studying SNRs. This evidence is not totally conclusive in all particulars, however. The modelling of the SSS data requires a complete time-dependent coupled solution of the hydrodynamics and ionization, including whatever density inhomogeneities may exist in each specific remnant, since the experiment aperture is 6 min in diameter. Better spectral resolution than its $160 \mathrm{eV}$ FWHM would be useful (insofar as K-emission contributions from differing multiplet components would then be resolvable), but the present resolution is sufficient to distinguish $\mathrm{H}-\mathrm{like}$ and He-like ionization states. Much more important would be the application of the available spectral resolution to much more localized regions of the SNR, so that differential information would not have to be extracted from the attempted separation into components of the whole-remnant spectrum.

The available procedure is necessarily non-unique, and cannot unambiguously distinguish whether the electrons in the recently shocked plasma can quickly equilibrate with the heavier constituents (as it least some fraction must, from > $10 \mathrm{keV}$ measurements; Pravdo and Smith 1979) or whether they require a Coulomb equilibration timescale (typically longer than the remnant age) to do so; the two cases yield very different bremsstrahlung continua and, hence, can result in very different blast/ejecta proportions and abundances. Unfortunately, fits to the whole remnant data with either assumption for the electron equilibration timescale can yield results of comparable statistical significance. With no independent reason for fixing such otherwise indeterminate parameters, therefore, model uniqueness is not assured. Intil such time as more independent information exists (e.q. from the application of like quality spectral data with much finer spatial resolution, so that some separability of otherwise degenerate fitting parameters can be obtained), a satisfactory fit to the whole-remnant spectral data cannot guarantee a unique detailed explanation.

My thanks to the many members and associates of the GSFC X-ray group, past and present, who have contributed to our ongoing program of research. Andrew Szymkowiak, in particular, is responsible for the large fraction of the previously unpublished work on which much of this discussion is based.

\section{REFERENCES}

Becker, R.H., Holt, S.S., Smith, B.W., White, N.E., Boldt, E.A., Mushotzky, R.F., and Serlemitsos, P.J. 1979, Ap. J. (Letters) 234, L73. 
Becker, R.H., Holt, S.S., Smith, B.W., White, N.E., Boldt, E.A., Mushotzky, R.F., and Serlemitsos, P.J. 1980a, Ap. J. (Letters) 235, L5.

Becker, R.H., Boldt, E.A., Holt, S.S., Serlemitsos, P.J., and White, N.E. 1980b, Ap. J. (Letters) 237, L77.

Becker, R.H, Szymkowiak, A.E., Boldt, E.A., Holt, S.S., and Serlemitsos, P.J. 1980c, Ap. J. (Letters) 240, L33.

Bowyer, C.S., Byram, E.T., Chubb, T.A., and Friedman, H. 1964, Science $146,912$.

Brisken, A.F. 1973, PH.D. Thesis (University of Maryland).

Charles, P.A., Culhane, J.L., Zarnecki, J.C.., and Fabian, A.C. 1975, Ao. J. (Letters) 197, L61.

Chevalier, R.A. 1982, Ap. J. 258, 790.

Davidson, K., Pacini, F. and Salpeter, E.E. 1971, Ap. J. 168, 45.

Davison, P.J.N., Culhane, J.L., and Mitche11, R.J. 1976, Ap. J. (Letters) 206, L37.

Fabian, A.C., Stewart, G.C., and Brinkmann, W. 1982, Nature 295, 508.

Fabian, A.C., Willingale, R., Pye, J.P., Murray, S.S., and Fabbiano, G. 1980, Mon. Not. R. Astron. Soc. 193, 175.

Gorenstein, P., Kellogg, E.M., and Gursky, H. 1970, Ap. J. 160, 199. Hamilton, A.J.S. 1983, this volume, p. 113.

Hamilton, A.J.S., Sarazin, C.L., and Chevalier, R.A. 1983, Ap. J. Suppl., in the press.

Heiles, C. 1964, Ap. J. 140, 470.

Hi11, R.W., Burginyon, G.A., and Seward, F.D. 1975, Ap. J. 200, 158. Holt, S.S., White, N.E., Becker, R.H., Boldt, E.A., Mushotzky, R.F., Serlemitsos, P.J., and Smith, B.W. 1979, Ap. J. (Letters) 234, L65. Itoh, H. 1977, Pub1. Astron. Soc. Japan 29, 813.

Pacini, F. 1971, Ap. J. (Letters) 163, LTT.

Pravdo, S.H., Becker, R.H., Boldt, E.A., Holt, S.S., Rothschild, R.E., Serlemitsos, P.J., and Swank, J.H. 1976, Ap. J. (Letters) 206, L41.

Pravdo, S.H. and Smith, B.W. 1979, Ap. J. (Letters) 234, L195.

Pravdo, S.H. 1983, this volume, n. 29.

Reynolds, S.P. and Chavalier, R.A. 1981, Ap. J. 245, 912.

Sarazin, C.L., Hamilton, A.J.S., and Chevalier, R.A. 1983, this volume, p. 102 .

Serlemitsos, P.J., Boldt, E.A., Holt, S.S., Ramaty, R., and Brisken, A.F. 1973, Ap. J. (Letters) 184, 1.

Seward, F., Gorenstein, P., and Tucker, W. 1983

Shk lovsky, I.S. 1967, Ap. J. (Letters) 148, L1.

Shu11, J.M. 1982, Ap. J. (Letters), in the press.

Woltjer, L. 1972, Ann. Rev. Astron. Ap. 10, 129.

\section{DISCUSSION}

KIRSHNER: The principal optical result on Cas $A$ is that oxygen is greatly enhanced. The SSS spectra only give the ratio of Si, S and the rest to oxygen. Are the $X$-ray data consistent with the extraordinary abundances seen in the optical filaments?

HOLT: Because the energy resolution of the SSS does not allow us to completely separate line and continuum contributions to the experimental spectrum, and because the bremsstrahlung continuum is dominated by 
oxygen (if present in at least solar proportions to hydrogen), it is not possible to define a totally unambiguous reference for the "abundances"--even before addressing the problem of how appropriate an equilibrium model should be for the whole-remnant spectrum of Cas $A$. If we assume that the portion of the spectrum below $\sim 3 \mathrm{keV}$ is dominated by the ejecta in the fast-moving knots, the implied Si/0 ratio is well within a factor of two of the values given in Table 5 of Chevalier and Kirschner (1978).

TUFFS: Is a shock velocity of, say, $2800 \mathrm{~km} \mathrm{~s}^{-1}$ for Cas A consistent with the observed line ratios?

HOLT: The shock temperature defined by the shock velocity (for a Sedov solution) is $>10^{8} \mathrm{~K}$, or much too high to match the observed line ratios. The experimental preponderance of helium-like to hydrogen-like lines forces the equilibrium fits to the spectra of Tycho and Kepler, in addition to that of Cas $A$, to contain a component at $<10^{7} \mathrm{~K}$ which dominates below $\sim 3 \mathrm{keV}$. At higher energies, however, there is continuum evidence for plasma approaching equilibrium with the blast wave (Pravdo and Smith 1979).

CHEVALIER: With regard to the non-uniqueness of the $X$-ray spectral fits, do all the models have the proper ages, radii and $X$-ray luminosities?

HOLT: The Sedov models we have attempted to fit to Tycho and Kepler, such as that displayed in Figure 3 for Kepler, are those for which the ages are correct and the radii/luminosities are consistent with reasonable distance estimates. We don't take seriously any fortuitous spectral fits with unreasonable model implications.

FEDORENKO: Are the power-law $X$-ray spectra consistent with the equilibrium particle distribution functions? What is the mechanism of radiation?

HOLT: I presume that you are referring to the spectrum of SN1006, for which we find that a power-law is an adequate fit to our data. It is certainly possible to synthesize power-law fits from combinations of equilibrium particle distribution functions (see the early attempt by Sartori and Morrison 1967 to so reproduce the spectrum of the Crab), but the consistency of the same power law over two orders of magnitude in energy is suggestive of a synchrotron origin for the $X$-ray emission.

FABIAN: Since the electron-ion equilibration time is often greater than the age of the remnant, how do you know what high energy spectral form should be fit to the continuum data (e.g. in SN1006)?

HOLT: In the case of Sedov models, we utilize computer-generated differential spectra instead of limiting analytic forms, which are dependent upon the usual model parameters (including time) as well as upon physical assumptions such as whether electron-ion equilibration 
can be more rapid than specified by Coulomb interactions alone. Although a power-law appearance is certainly possible for the superposition of non-equilibrium as well as equilibrium thermal spectra, the extension of the form to at least $20 \mathrm{keV}$ for SN1006 argues against it because such a hard spectrum $(\alpha \sim 1)$ suggests a blast wave with much higher velocity than that of the younger and more energetic Cas $A$, for example (with $\alpha \sim 3$ ); the appearance of any photons at all at $20 \mathrm{keV}$ requires a current blast wave velocity of $4000 \mathrm{~km} \mathrm{~s}^{-1}$, but the maintenance of the hard spectral form requires that the blast wave velocity is considerably larger than that. The assumption of a slow equilibration time only exacerbates the problem, as it is then even more difficult to produce X-rays with energies comparable to the value for equilibrium with the blast wave and, hence, more difficult to produce both a hard spectrum and its extension out to $20 \mathrm{keV}$. 